\title{
Structural imaging of the pancreas in rat using micro-CT: application to a non-invasive longitudinal evaluation of pancreatic ductal carcinoma monitoring
}

\author{
Akladios $\mathrm{CY}^{1, *}$, Bour $\mathrm{G}^{1}$, Raykov Z $\mathrm{Z}^{2}$, Mutter $\mathrm{D}^{1}$, Marescaux $\mathrm{J}^{1}$ and Aprahamian $\mathrm{M}^{2}$ \\ ${ }^{1}$ Institut de Recherche contre les Cancers de l'Appareil Digestif (IRCAD), 1 place de l'hôpital, 67000 Strasbourg, France \\ ${ }^{2}$ Institut National pour la Santé et la Recherche Médicale, Unit 701, IRCAD, 1 place de l’hôpital, 67000 Strasbourg, France
}

\begin{abstract}
The aim of the study was to evaluate the feasibility of a longitudinal non-invasive monitoring of rat pancreatic ductal adenocarcinoma (PDAC) using microCTscans $(\mu \mathrm{CT})$. The identification of the pancreatic gland on $(\mu \mathrm{CT})$ was performed at first using contrast products (Fenestra LC and VC, v/v) at a dosage of $0.5 \mathrm{ml} / \mathrm{Kg}$ of body weight. Then orthotopic PDAC developed in adult Lewis rat was detected and monitored. In vivo $\mu$ CT measurement of tumor was compared to actual size ex vivo in 12 rats. Gemcitabine treatment of PDAC was monitored at two week intervals until defined endpoints (liver metastasis or ascitis) in 10 rats versus 10 controls. $\mu$ CT had a $100 \%$ positive predictive value in the detection of orthotropic PDAC. Regression analysis showed a linear correlation between ex vivo and in vivo $\mu \mathrm{CT}$ tumor measurements. Longitudinal evaluation of tumor progression showed a reduction in tumor growth $(\mathrm{P}<0.05$ at 8 weeks) and a slightly prolonged survival $(\mathrm{P}=0.15)$ under gemcitabine treatment. In conclusion $\mu \mathrm{CT}$ appears to be a cost-effective mean for preclinical study of PDAC saving time, animals, while respecting animal welfare. It could be considered as an efficient tool in anticancer drug research and development.
\end{abstract}

Keywords: microCT; orthotopic tumor model; pancreatic adenocarcinoma; rat

\section{Introduction}

In Europe pancreatic ductal adenocarcinoma has an incidence of approximately 10 per 100,000 populations per year. Unfortunately, the numbers for incidence and mortality are still practically identical for this cancer which signifies how devastating is its prognosis [1]. Progress in treatment options of this deadly disease has still not been achieved. Thus, animal models are crucially needed for better understanding of its pathogenesis and to explore new potential therapeutic targets [2].

Classically, the detection of orthotopic, chemically-induced or genetic models of tumors or the monitoring of their development as the evaluation of emerging therapeutics, needs animal sacrifice to obtain a single accurate measurement of tumor size. This is time, animal and money consuming. Structural imaging technologies such as micro-computed tomography $(\mu \mathrm{CT})$ have the potential to become invaluable tools in this tumoral evaluation. Besides being a non-invasive method of follow up, it will increase data reliability as each animal will remain its own control all over the longitudinal evaluation of tumor evolution, reducing the number of animals needed for confidence.
Computed tomography constitutes the current "standard" for evaluation of tumor size and spread in clinical setting because of its undeniable superiority compared to simple palpation estimate [3]. As a result, radiologic evaluation of the tumors during clinical trials of anticancer pharmaceuticals has evolved into a surrogate marker of therapeutic response [4]. However, at present implementation of sequential $\mu \mathrm{CT}$ as a measurement

*Corresponding author: Akladios CY, Institut de Recherche contre les Cancers de l'Appareil Digestif (IRCAD), 1 place de l'hôpital, 67000 Strasbourg, France, Tel.: +33 6648420 63; Fax: +33 3881274 57; E-mail: cherif.youssef2@wanadoo.fr

Received 12 December 2012 Revised 5 February 2013 Accepted 12 February 2013 Published 19 February 2013

Citation: Akladios CY, Bour G, Raykov Z, Mutter D, Marescaux J, Aprahamian M (2013) Structural imaging of the pancreas in rat using micro-CT: application to a non-invasive longitudinal evaluation of pancreatic ductal carcinoma monitoring. J Cancer Res Ther 1: 70-76. doi:10.14312/2052-4994.2013-11

Copyright: (C) 2013 Akladios CY, et al. This is an open-access article distributed under the terms of the Creative Commons Attribution License, which permits unrestricted use, distribution and reproduction in any medium, provided the original author and source are credited. 
tool remains still relatively rare in small animal studies [5-15]. Furthermore until now, very few data concerning its use for pancreatic imaging in animal models have been reported in the literature [16].

The present study aimed to evaluate at first the reliability of $\mu \mathrm{CT}$ in identification of normal pancreas in a rat model, then pancreases bearing an orthotopic pancreatic ductal adenocarcinoma (PDAC). Tumor detection and tumor size determination were subsequently assessed. Afterwards the potential of $\mu \mathrm{CT}$ for longitudinal evaluation of tumor evolution was investigated in this tumor model through its actual monitoring under conventional chemotherapy for PDAC (gemcitabine).

\section{Materials and methods}

\section{Animals and orthotopic tumor model}

All animal experiments were performed according to the directives of the European Community Council (STE number 123 of June 15, 2006) for animal use and care. Thirty two Lewis rats (Janvier, Le Genest Saint Isle, France) (7 weeks old; minimum weight $180 \mathrm{~g}$ ) were used for this experiment. Implantation of the ductal tumor cells [17] was performed in the tail of the pancreas under laparotomy as described by Mutter et al. [18]. Surgical and imaging procedures were performed on rats breathing a mixture of $3 \%$ isoflurane (Forene $®$, Abbott, Rungis, France) in pure $\mathrm{O}_{2}$. Analgesia was achieved by a concomitant intramuscular injection of xylazine hydrochloride (Rompun $®$, Bayer, Leverkusen, Germany, $2 \mathrm{mg} / \mathrm{kg}$ ) for surgery. The endpoints for survival experiments consisted in either clinical evidence of animal suffering (e.g. prostration, bad-grooming, abdominal distension consistent with a hemorrhagic ascitis, anaemia revealed by conjonctival and mucosal bleaching) or imaging evidence of ascitis and presence of liver metastasis.

\section{Image acquisition and reconstruction}

The images were acquired at approximately $10 \mathrm{~min}$ on the microCAT-II (Siemens Preclinical Solutions, Knoxville, $\mathrm{TN}$ ) at $80 \mathrm{kVp}$ with an anode current of $500 \mu \mathrm{A}$, under respiratory-gated acquisition of free breathing animals [8]. Scans were completed over $360^{\circ}$ of rotation with 361 projections. The reconstructions were performed using a filtered back-projection algorithm (Cobra, Version 4.1-4, Exxim, Knoxville, TN) with a slice thickness of $100 \mu \mathrm{m}$. Final 3D data visualized with the Amira software (Version 3.1, TGS, San Diego, CA) provided an image with a size of 512 × 512 × 416 voxels (e.g. a cube with a side of $6.7 \mathrm{~cm}$, as voxel size was $131 \times 131 \times 162 \mu \mathrm{m}$ ).

\section{Dosage and kinetics of enhanced-contrast agents}

According to the manufacturer recommends, the ITG contrast enhancers for $\mu \mathrm{CT}$, trade-named Fenestra ${ }^{\circledR}$ liver contrast (LC) and vascular contrast (VC) both from Advanced Research Technologies (Inc., Montreal, Canada), [9-11] should be injected together intravenously (vol/vol) at the dose of $1 \mathrm{ml} / 100 \mathrm{~g}$ of body weight for each of them, with a iodine concentration of $50 \mathrm{mg} / \mathrm{ml}$. Such a fluid volume highly exceeds the maximal intravenous amount allowed by the European current best practice guideline, which is $0.5 \mathrm{ml} / 100 \mathrm{~g}$ body weight at the maximum corresponding to $10 \%$ of the rat volemia. In this way, the maximal allowed dosage of contrast agent corresponds to 4 times less than the recommended amount. So in order to overcome this limitation, we set up a strategy using two different routes: first intraperitoneal and then intravenous administration for a total dosage of $0.5 \mathrm{ml} / 100 \mathrm{~g}$.

Half of this dose was administrated as an LC product by an intraperitonealy $6 \mathrm{~h}$ before, and then the second half as VC product intravenously just before imaging, according to a procedure previously described [19]. Such a protocol allows contrast enhancement of abdominal organs (such as liver, gut, spleen and kidneys) and vessels allowing recognition of the pancreatic gland by the identification of its vessels.

\section{Confirmation of pancreatic tumor measurement accuracy with CT scan}

To confirm the validity of tumor measurements by CT scan [7] as far as PDAC is concerned, $\mu$ CT imaging was carried out on 12 Lewis rats bearing an orthotopic PDAC from the second to the sixth week after inoculation. Final 3D data visualized with the Amira software showed the tumors as a non-contrasted area within the pancreas. Determination of in vivo and ex vivo tumor volume was performed by the same operator. The results are the mean of 3 different measurements in both situations. Tumor volumes were computed from three-dimensional measurements performed in coronal, sagittal and axial planes, according to the formula of a hemi ellipsoid ( $\pi . X . Y . Z / 6)$. The individual actual size of the genuine PDAC (confirmed by histological analysis) was established at autopsy of animals included in the study, by caliper measurement of the dissected tumor. The resulting ex vivo volumes were compared with their corresponding in vivo measurement.

\section{Longitudinal evaluation of pancreatic tumor evolution}

Twenty Lewis rats carrying PDAC were used for assessments of $\mu \mathrm{CT}$ ability to perform reliable follow up and the behavior of this tumor. An initial determination of tumor size was carried two weeks after implantation in the pancreatic tail, as previously determined [18]. Then animals were randomly affected to either a control group $(n=10)$ without any treatment or a group treated by conventional chemotherapy $(n=10)$ with gemcitabine (Gemzar, Lilly France, Fegersheim, France). Animals received, as previously described [16], two consecutive injections of $50 \mathrm{mg} / \mathrm{Kg}$ of Gemzar at days 14 and 16 post tumor implantation corresponding to a total of 100 $\mathrm{mg} / \mathrm{Kg}$. Micro-CT imaging was performed at two week intervals for determination of tumor volume as long as six animals remained alive in each experimental group. 


\section{Statistical analysis}

A potential difference between the data of the in vivo and ex vivo size measurement was tested using a one-way analysis of variance followed by a parametric Student's paired t-test, Bartlett's test indicating homogeneity of variance. The difference between the values was considered significant when $\mathrm{P}<0.05$. Correlations between gross and CT determinations of tumor volume were tested using a parametric Pearson test, as variances were not significantly different. The correlation was considered significant when $\mathrm{P}<0.05$. Potential difference in tumor volume evolution resulting from chemotherapy was tested by a non-parametric Mann Whitney test as Bartlett's test indicating heterogeneity of variance. The survival curves were generated using the Kaplan-Meier method and the differences between the curves were assessed by the log-rank test. A P-value $<0.05$ was considered to be statistically significant. Instat 2.00 Macintosh software (GraphPad Software, San Diego, CA) was used.

\section{Results}

Pancreatic and tumor recognition after contrast enhancement

The pancreas can be visualized using a two-step injection of at first the hepatic contrast agent intraperitonealy $6 \mathrm{~h}$ before followed by an equal dose of vascular enhancer just before imaging. $\mu \mathrm{CT}$ imaging after contrast administration allows a clear identification of the pancreas among the well-contrasted surrounding organs (Figure 1). It looks like a flat structure expanded on coronal slices (Figure 1a) between the duodenum loop and the spleen (delineated by dotted curves). The same aspect delineated by dotted curves is observed on axial slices (Figure 1b). The pancreas, situated in dorsal position behind the stomach, is elongated between the right lateral lobe of the liver and the spleen. On sagittal slices (Figure 1c), the corpus of the gland is in close contact with the dorsal face of the stomach and the apical pole of the kidney (dotted curves)

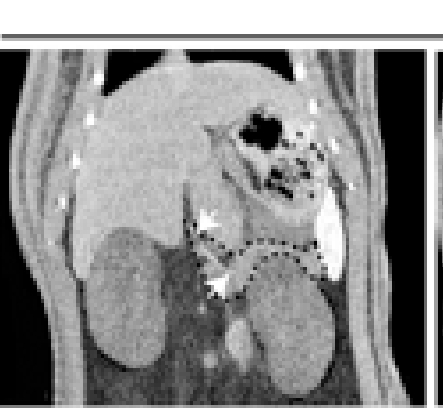

Normal pancreas
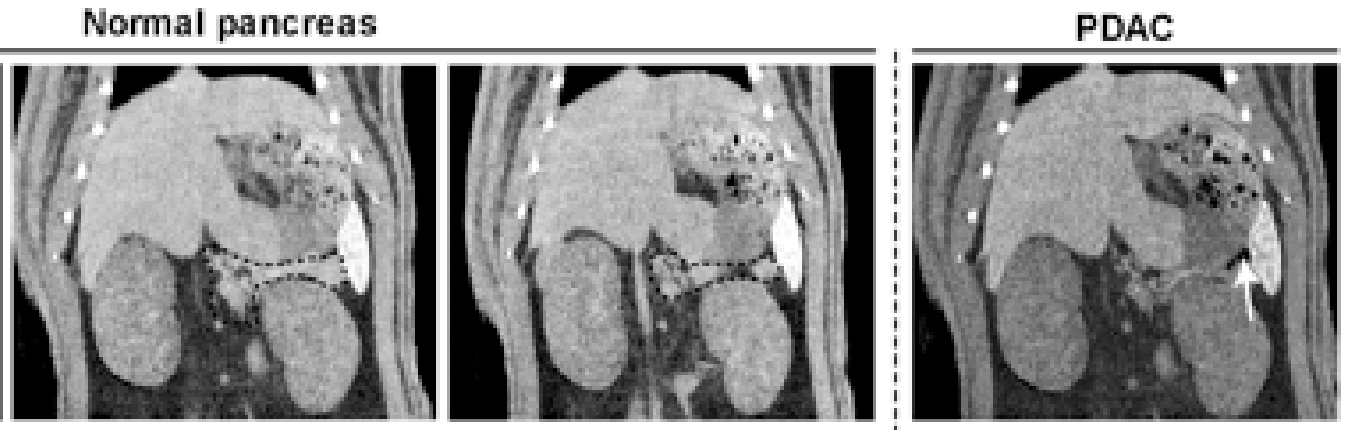

(a)
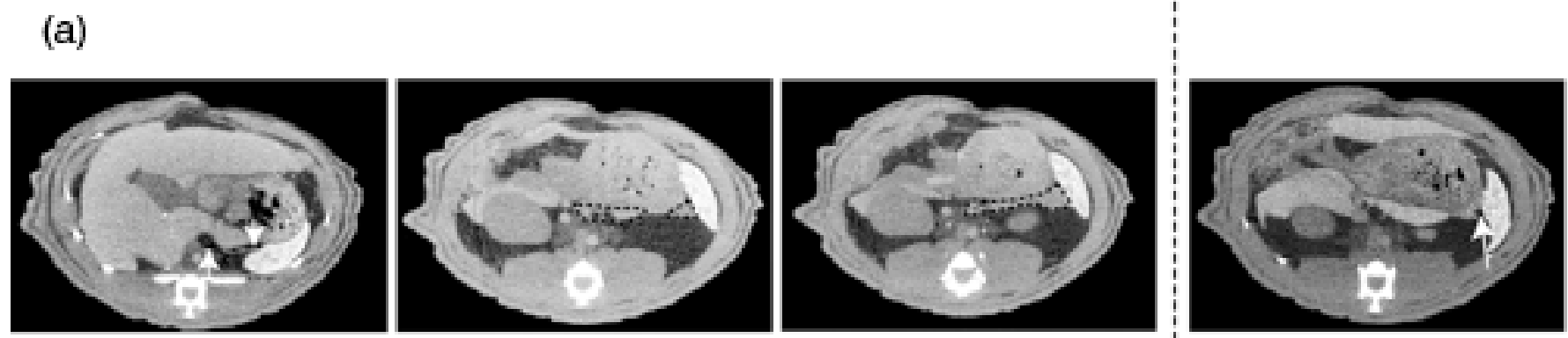

(b)
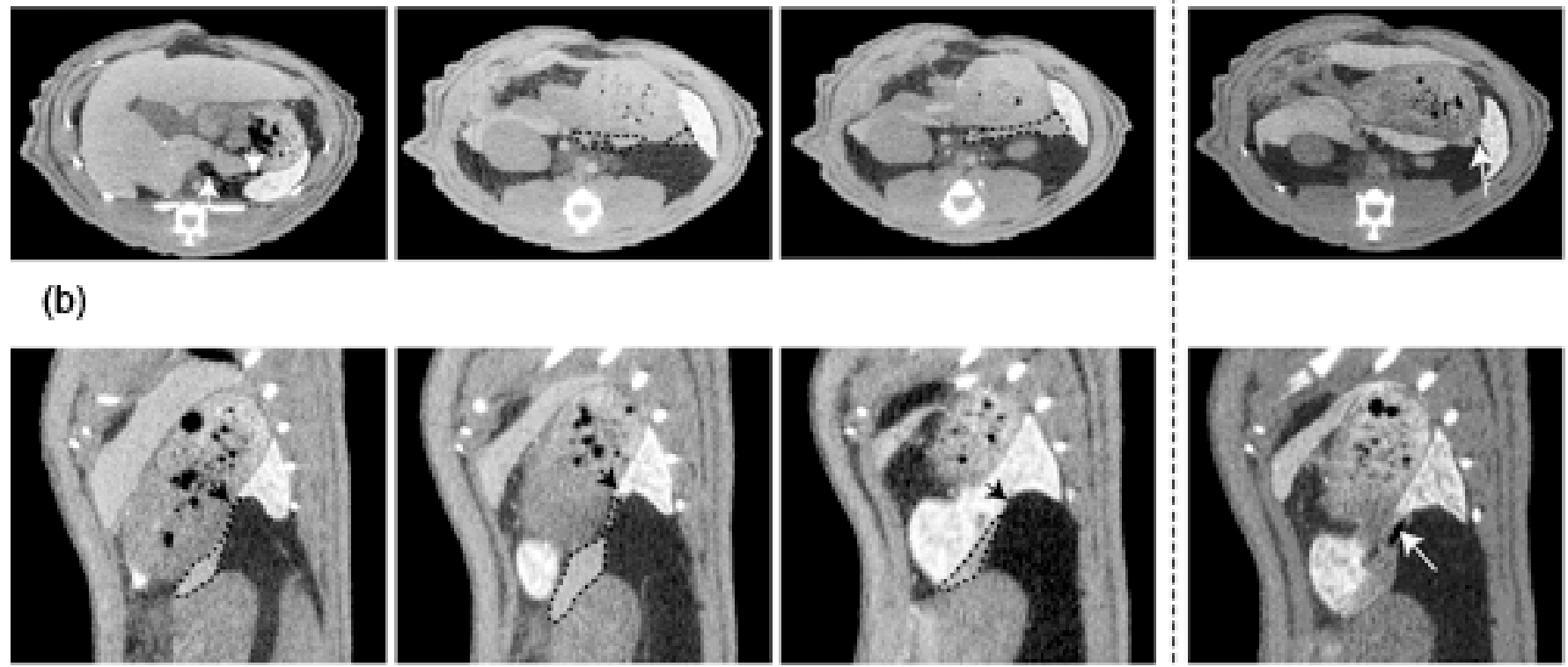

(c)

Figure 1 Structural imaging of pancreas allows diagnosis of small sized tumors. (a) Representative coronal sections of the pancreas without and with a small tumor in the tail. The pancreatic gland, including head, corpus and tail was outlined by a black dotted curve. The splenic vein (left panel) drained by the portal vein was indicated by two white arrows. A small sized PDAC (right panel), seated in the pancreatic tail, can be visualized as a small defect (white arrow). (b) Axial sections of the pancreas without and with a small tumor in the tail. The pancreas was outlined by a black dotted curve. The splenic artery (left panel), coming from the abdominal aorta, was indicated by two white arrows. The small PDAC (right panel) was also indicated by a white arrow. (c) Sagittal sections of the pancreas without and with a small tumor in the tail. The tail of the pancreas, connected to the porta of the spleen (black arrows), was outlined by a black dotted curve. The small PDAC (right panel) was indicated by a white arrow. 
and the tail is attached to the porta of the spleen (black arrows). The dorsal wall of the pancreas is marked by the splenic artery (left panel of Figure 1b, white arrows) and vein (left panel of Figure 1a, white arrows). Identification of these vessels contributes in pancreas recognition. Tumors, initiated in the tail, were identified two weeks later close to the spleen (white arrows in the right panels of Figure 1) under the aspect of small black holes (0.05$00.1 \mathrm{~cm}^{3}$ in size) in the enhanced pancreatic parenchyma. Their tumoral nature was confirmed at autopsy.

\section{$\mu C T$ allows tumor diagnosis and accurate volume determination}

The individual actual size of the genuine PDAC tumors established by caliper measurement at the autopsy of the animals included in the study $(n=12)$ were compared with their corresponding in vivo determinations. A nearly perfect linear correlation $(\mathrm{R} 2=0.986, \mathrm{P}<0.001)$ was observed between the two methods (Figure 2a). There was no case of false negative or positive tumor recognition with $\mu \mathrm{CT}$, even at the earlier time ( 2 weeks) when tumors volumes are still small. H\&E-stained tumor sections (Figure 2b) showed a well-differentiated carcinoma with some mitotic figures and a ductal pattern.

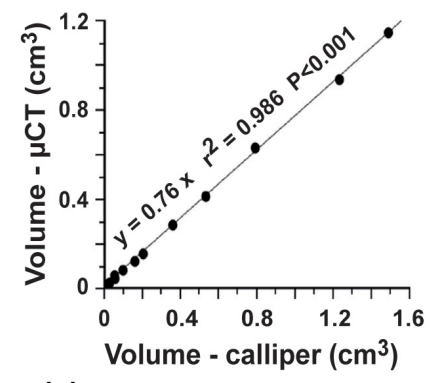

(a)

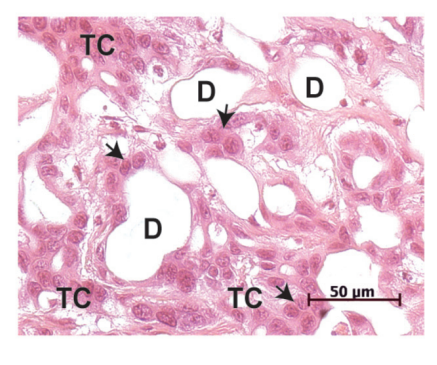

(b)
Figure 2 (a) Correlation between non-invasive quantification of tumor volumes with $\mu \mathrm{CT}$ and actual measurements of the same tumors at autopsy with caliper measurements. Tumor detection started at 10-14 days after implantation with a volume of $25 \mathrm{~mm} 3$. (b) Photomicrograph of a hematoxylin-eosin stained section of Lewis pancreatic tumor. The pancreatic tumor cells (TC), organized in an obvious ductal structure with numerous ducts (D) look cuboidal (black arrows) with minimal nuclear polymorphism.

\section{Longitudinal evaluation of PDAC revealed a poor efficiency of gemcitabine treatment}

Tumors were monitored on 20 Lewis rats bearing an orthotopic PDAC assessed by $\mu$ CT 2 weeks after induction, as illustrated in Figure 3a (the tumor is delineated by a white dotted curve). Monitoring of PDAC growth was then performed by recurrent $\mu$ CTs at two week intervals. The pancreatic tail was fully invaded between 6 and 8 weeks, the tumor pressing on the surrounding organs, making its way to local invasion (white arrows in right panel) and metastasis. At this stage, animals were euthanazied as soon as their CT scans revealed the occurrence of one of the experimental endpoints illustrated in Figure $3 \mathrm{~b}$. These end points are at first the evidence of local extension (white arrows) involving stomach and gut, then of an ascitis disjointing liver lobes with a liquid lamina, and/or liver metastasis with vessel stricture.

The potential for preclinical pharmacological studies of the longitudinal evaluation of tumor progression by imaging was tested in 10 rats treated with gemcitabine by comparison with 10 controls. Tumor volumes progression was monitored by recurrent $\mu \mathrm{CT}$ at two week intervals over the survival period. Volumes were calculated for all animals and their respective means were statistically compared and plotted for each time point of investigation as long as 6 animals per experimental group remained. Such a monitoring of PDAC revealed a significant retardation in tumor growth (Figure 3c) and a slight improvement in median survival $(P=0.071)$ as in actual survival $(P=0.149)$ using gemcitabine chemotherapy (Figure 3d).

\section{Discussion}

Structural imaging of visceral organs of small animals offers currently the ability to perform a longitudinal evaluation of their pathologies and to carry out conclusive preclinical studies on a potential therapeutic benefit. This emerging experimental method allows to explore transgenic mice, in which spontaneously arising tumor develop at unpredictable times, as orthotopically implanted tumor models to perform size monitoring over time without animal sacrifice. Besides improving the animal use and welfare, structural imaging increases experimental safety, as each animal, monitored over time, can provide tumor dimensions over multiple time points. Since the animal need not be sacrificed in order to measure the tumor, the number of animals needed for any given trial would be reduced.

Small animal imaging technologies have been already extensively applied for liver imaging using $\mu \mathrm{CT}$ or MRI [20-22] at a lesser degree, but not for pancreas. Few MRI [23-25] and one $\mu$ CT studies [16] for pancreatic tumor detection in mice are available in the literature. The purpose of the present study was to provide a first description of the $\mu \mathrm{CT}$ aspect of normal pancreas in rats, and then to illustrate the $\mu \mathrm{CT}$ imaging potential for longitudinal evaluation of PDAC under a given chemotherapy. Although the use of rats in orthotopic cancer research is declining related to the availability of tumor model arising spontaneously in transgenic mice, such feasibility study of normal pancreas imaging and pancreatic tumor follow up was easily achieved in the rats due to the spatial resolution of the used $\mu \mathrm{CT}$ scan. A concern for this purpose was the deleterious effects of current $\mu \mathrm{CT}$ contrast enhancers [26]. Their recommended dosages $[5,7,8]$ provide well-enhanced imaging of the abdominal content, but were found to be too high, $[19,26,27]$ inducing systemic overload more or less responsible for pulmonary oedema [28] and heart failure [29]. Moreover, in our hands [19] these contrast enhancers, containing iodinated triglycerides $[5,7,8$, 

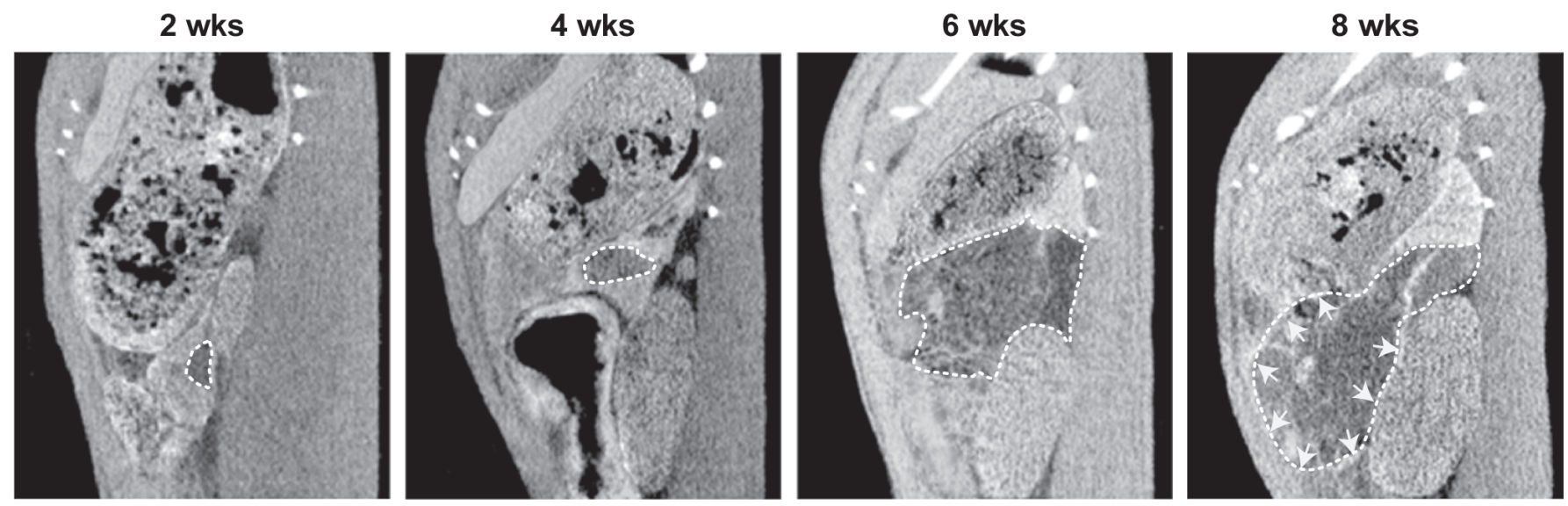

(a)
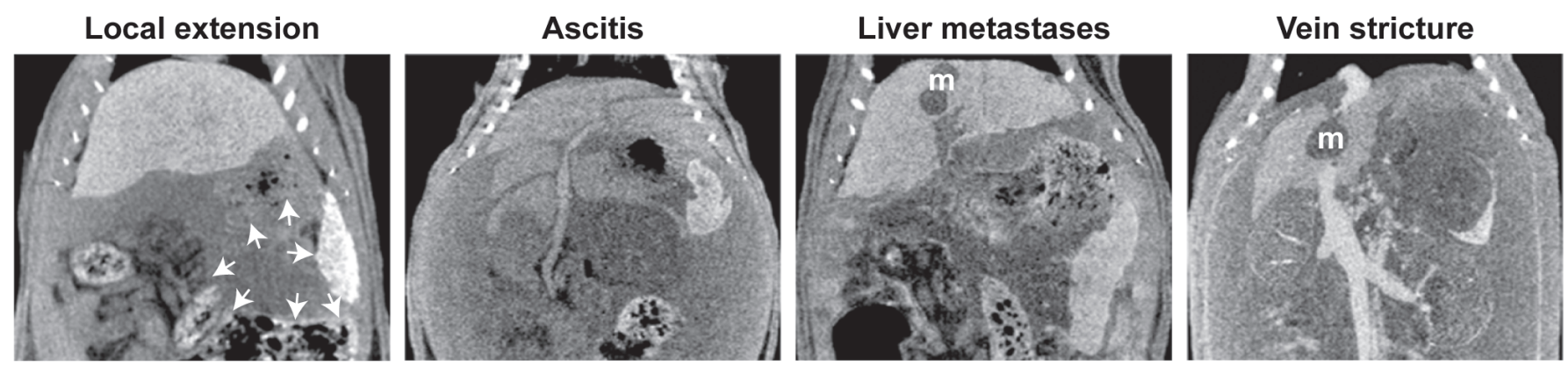

(b)

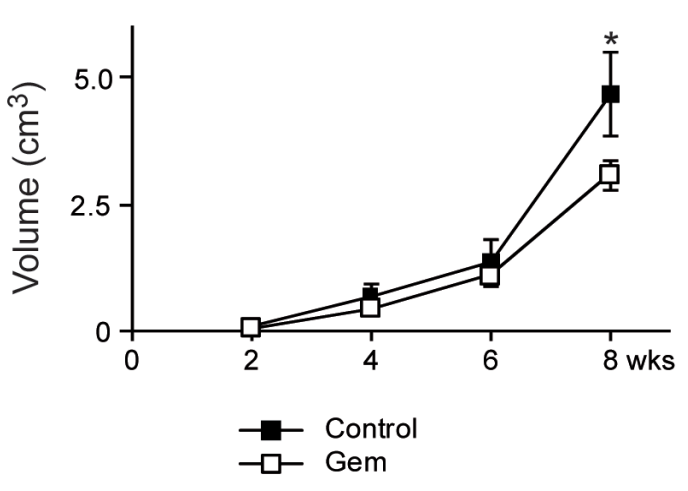

(c)

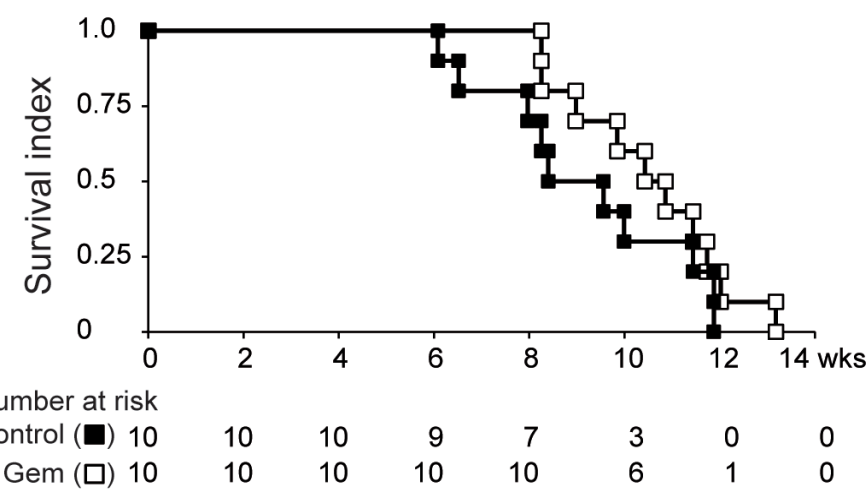

(d)

Figure 3 Longitudinal evaluation of PDAC revealed the efficiency of gemcitabine treatment. (a) Representative pattern of PDAC growth provided by recurrent $\mu \mathrm{CT}$ on a single control animal. Two weeks after induction, $\mu \mathrm{CT}$ assessed the presence of a tumor, seated in the pancreatic tail. The tumor, outlined by a white dotted curve, filled progressively the whole pancreatic tail, forcing back and then invading neighboring viscera. Local invasion (left panel) is indicated by white arrows. (b) Representative coronal slices illustrating the imaging experimental end points for PDAC: local progression with extension towards spleen, caecum, colon and stomach (white arrows), hemorrhagic ascitis with abdominal distension and separation of liver in distinct lobes, liver metastases (m) and vein stricture by the metastatic process. (c) Evolution of tumor size in animals treated with gemcitabine (100 $\mathrm{mg} / \mathrm{kg}$ in two injections at 2-day interval) versus controls. A significant difference was observed in terms of tumor growth after six weeks of experiment $\left({ }^{*}<0.05\right.$, Student $\mathrm{t}$-test). Data are means $( \pm \mathrm{sem})$ of individual values as a function of time $(\mathrm{n}=10$, except for the last volume determination). (d) Survival of gemcitabine-treated animals versus controls. Ten rats of each group were monitored over 82 days, at which time the experiment was terminated, all animals reaching experimental endpoints. Animal survival is represented as Kaplan-Meier curves. No significant difference was found between actual survivals.

26] induces a failure in liver functions consistent with biological disturbance (increased serum transaminases and bilirubin) and clinical jaundice (yellowish staining of the sclera and emission of brown urines). A first set of experiments has determined the dose, the composition and the route of administration of contrast enhanced adapted to animal welfare and providing a satisfying organ and vessel enhancement [19]. Moreover, this twostep strategy, associating the peritoneal and vascular routes resulted in an optimal accuracy in tumor detection in our study with no false negative readings even for very small pancreatic tumors. Even though CT imagingbased methods have been validated on mammary tumors to assess tumor response instead of traditional caliper- 
based measurements [7], we need however to confirm its validity on PDAC, for which no similar data are available.

This accuracy in small tumor detection $[5,7,8,10,14$, $15,22]$ is an advantage of using $\mu$ CT [22] for longitudinal evaluation of tumors. However, MRI using magnetic fields is assumed to be a harmless imaging procedure [20-25] even if newly controversial, $[30,31]$ whilst $\mu \mathrm{CT}$, an X-raybased approach, raises concerns about immunological and general physiological changes that might affect the experimental outcome [32]. Indeed, if the irradiation level (2.5 cGy for 10 min acquisition with an Imtek $\mu \mathrm{CT}$ in rats) [33] is notably lower than sub-lethal doses [14, $33,34]$, it can transiently damage the support of genetic information [35]. It is well known however from clinical trials that PDAC is extremely radioresistant [36] and the analysis of clinical features of our experiment revealed that the growth of the experimental PDAC was not affected by recurrent $\mu \mathrm{CT}$ imaging [18].

As already observed, rodent pancreas has been poorly investigated using non-invasive imaging methods in vivo. MRI studies using gadolinium described pancreatic tumor detection as well as liver metastasis follow up in mice [23-25]. Visualization of the pancreas, which is in rodents a flat and thin lobular gland expended between the duodenum and spleen, needs serial thin adjacent slices of the upper part of the abdomen (1-mm thickness or less). A careful screening of these slices, sustained by a good knowledge of rodent abdominal anatomy, allows its identification. This identification is helped by the recognition of splenic vessels delineating the pancreatic edges. Then, the detection of even a very small pancreatic tumor (as low as $2 \mathrm{~mm}$ dimension) becomes possible, allowing its management from an early stage.

The longitudinal evaluation of tumor progression through $\mu \mathrm{CT}$ is carried out without sacrifice at two week intervals and thereby uses a smaller group of experimental animals. It can be also possible to express tumor progression as relative changes of the initial volume at 2 weeks, each animal constitutes thus his own control of tumor progression throughout the experiment. The power of this methodology was demonstrated in the current study on PDAC, by the longitudinal evaluation of conventional gemicitabine chemotherapy. The ability of non-invasive imaging to provide new criteria for experiment endpoints is also of interest. Imaging endpoint for PDAC as early signs of ascitis, liver metastasis, and vessel or bowel strictures can be easily determined. These imaging features are detectable before any clinical evidence, anticipating the observance of animal pain that constitutes until yet the experimental endpoint.

\section{Conclusions}

In conclusion, $\mu \mathrm{CT}$ of animal models of pancreatic cancer is an emerging cost-effective mean of tumor monitoring, saving time and more particularly animal welfare and expand. It can be applied to the screening and comparison of new therapeutics, by providing valuable information on tumor growth in a single experimental set up.

\section{Conflict of interest}

All the authors declare that they have no conflict of interest.

\section{Acknowledgments}

Supported by the French Agence National pour la Recherche (grant ANR no. 06-BLAN-0141-0002), the Association pour la Recherche sur le Cancer (grant ARC no 3153-2007) and the European Research Council advanced Grant of JM Egly (2009).

\section{References}

[1] Krejs GJ (2010) Pancreatic cancer: epidemiology and risk factors. Dig Dis 28:355-358.

[2] Kalinina T, Güngör C, Thieltges S, Möller-Krull M, Penas EM, et al. (2010) Establishment and characterization of a new human pancreatic adenocarcinoma cell line with high metastatic potential to the lung. BMC Cancer 10:295.

[3] Prasad SR, Jhaveri KS, Saini S, Hahn PF, Halpern EF, et al. (2002) CT tumor measurement for therapeutic response assessment: comparison of unidimensional, bidimensional, and volumetric techniques initial observations. Radiology 225:416-419.

[4] Saini S (2001) Radiologic measurement of tumor size in clinical trials: past, present, and future. AJR Am J Roentgenol 176:333334.

[5] Weber SM, Peterson KA, Durkee B, Qi C, Longino M, et al. (2004 Imaging of murine liver tumor using microCT with a hepatocyteselective contrast agent: accuracy is dependent on adequate contrast enhancement. J Surg Res 119:41-45.

[6] Fournier LS, Cuenod CA, de Bazelaire C, Siauve N, Rosty C, et al. (2004) Early modifications of hepatic perfusion measured by functional CT in a rat model of hepatocellular carcinoma using a blood pool contrast agent. Eur Radiol 2125-2133.

[7] Ishimori T, Tatsumi M, Wahl RL (2005) Tumor response assessment is more robust with sequential CT scanning than external caliper measurements. Acad Radiol 12:776-781.

[8] Martiniova L, Schimel D, Lai EW, Limpuangthip A, Kvetnansky R, et al. (2010) In vivo micro-CT imaging of liver lesions in small animal models Methods 50:20-25.

[9] Weichert JP, Longino MA, Bakan DA, Spigarelli MG, Chou TS, et al. (1995) Polyiodinated triglyceride analogs as potential computed tomography imaging agents for the liver. J Med Chem 38:636-646.

[10] Weichert JP, Lee FT Jr, Chosy SG, Longino MA, Kuhlman JE, et al. (2000) Combined hepatocyte-selective and blood-pool contrast agents for the CT detection of experimental liver tumors in rabbits. Radiology 216:865-871.

[11] Bakan DA, Doerr-Stevens JK, Weichert JP, Longino MA, Lee FT Jr, et al. (2001) Imaging efficacy of a hepatocyte-selective polyiodinated triglyceride for contrast-enhanced computed tomography. Am J Ther 8:359-365.

[12] Li X, Zheng CS, Feng GS, Zhuo CK, Zhao JG, et al. (2002) An implantable rat liver tumor model for experimental transarterial chemoembolization therapy and its imaging features. World J Gastroenterol 8:1035-1039.

[13] Badea CT, Hedlund LW, De Lin M, Boslego Mackel JF, Johnson GA (2006) Tumor imaging in small animals with a combined microCT/micro-DSA system using iodinated conventional and blood pool contrast agents. Contrast Media Mol Imaging 1:153-164.

[14] Almajdub M, Nejjari M, Poncet G, Magnier L, Chereul E, et al. (2007) In-vivo high-resolution X-ray microtomography for liver and spleen tumor assessment in mice. Contrast Media Mol Imaging 2:88-93.

[15] Graham KC, Ford NL, MacKenzie LT, Postenka CO, Groom AC, et al. (2008) Noninvasive quantification of tumor volume in preclinical liver metastasis models using contrast-enhanced x-ray computed tomography. Invest Radiol 43:92-99. 
[16] Angelova AL, Aprahamian M, Grekova SP, Hajri A, Leuchs B, et al. (2009) Improvement of gemcitabine-based therapy of pancreatic carcinoma by means of oncolytic parvovirus H-1PV. Clin Cancer Res 15:511-519.

[17] Pettengill OS, Faris RA, Bell RH Jr, Kuhlmann ET, Longnecker DS (1993) Derivation of ductlike cell lines from a transplantable acinar cell carcinoma of the rat pancreas. Am J Pathol 143:292-303.

[18] Mutter D, Hajri A, Tassetti V, Solis-Caxaj C, Aprahamian M, et al. (1999) Increased tumor growth and spread after laparoscopy vs laparotomy: influence of tumor manipulation in a rat model. Surg Endosc 13:365-370.

[19] Akladios CY, Bour G, Balboni G, Mutter D, Marescaux J, et al. (2011) Contribution of microCT structural imaging to preclinical evaluation of hepatocellular carcinoma chemotherapeutics on orthotopic graft in ACI rats. Bull Cancer 98:120-132.

[20] Brockmann MA, Kemmling A, Groden C (2007) Current issues and perspectives in small rodent magnetic resonance imaging using clinical MRI scanners. Methods 43:79-87.

[21] Cheung JS, Guo H, Leung JC, Man K, Lai KN, et al. (2008) MRI visualization of rodent liver structure and peritoneal adhesion with dialyzate enhancement. Magn Reson Med 59:1170-1174.

[22] Martiniova L, Kotys MS, Thomasson D, Schimel D, Lai EW, et al. (2009) Noninvasive monitoring of a murine model of metastatic pheochromocytoma: a comparison of contrast-enhanced microCT and nonenhanced MRI. J Magn Reson Imaging 29:685-691.

[23] Grimm J, Potthast A, Wunder A, Moore A (2003) Magnetic resonance imaging of the pancreas and pancreatic tumors in a mouse orthotopic model of human cancer. Int J Cancer 106: 806-811.

[24] Montet X, Weissleder R, Josephson L (2006) Imaging pancreatic cancer with a peptide-nanoparticle conjugate targeted to normal pancreas. Bioconjug Chem 17:905-911.

[25] Partecke IL, Kaeding A, Sendler M, Albers N, Kühn JP, et al. (2011) In vivo imaging of pancreatic tumours and liver metastases using 7 Tesla MRI in a murine orthotopic pancreatic cancer model and a liver metastases model. BMC Cancer 28:11:40

[26] Hallouard F, Anton N, Choquet P, Constantinesco A, Vandamme T (2010) Iodinated blood pool contrast media for preclinical X-ray imaging applications--a review. Biomaterials 31:6249-6268.

[27] Morton DB, Jennings M, Buckwell A, Ewbank R, Godfrey C, et al. (2001 Refining procedures for the administration of substances. Report of the BVAAWF/FRAME/RSPCA/UFAW Joint working group on refinement. Lab Anim 35:1-41.

[28] Paré PD, Brooks LA, Baile EM (1981) Effect of systemic venous hypertension on pulmonary function and lung water. J Appl Physiol 51:592-597.

[29] Slutsky RA, Peck WW, Higgins CB (1983) Pulmonary edema formation with myocardial infarction and left atrial hypertension: intravascular and extravascular pulmonary fluid volumes. Circulation 68:164-169.

[30] Hasebroock KM, Serkova NJ (2009) Toxicity of MRI and CT contrast agents. Expert Opin Drug Metab Toxicol 5:403-416.

[31] Hartwig V, Giovannetti G, Vanello N, Lombardi M, Landini L, et al . (2009) Biological effects and safety in magnetic resonance imaging: a review. Int J Environ Res Public Health 6:1778-1798.

[32] Beckmann N, Kneuer R, Gremlich HU, Karmouty-Quintana H, Blé FX, et al. (2007) In vivo mouse imaging and spectroscopy in drug discovery. NMR Biomed 20:154-185.

[33] Boone JM, Velazquez O, Cherry SR (2004) Small-animal X-ray dose from micro-CT. Mol Imaging 3:149-158.

[34] Ford NL, Thornton MM, Holdsworth DW (2003) Fundamental image quality limits for microcomputed tomography in small animals. Med Phys 30:869-877.

[35] Löbrich M, Rief N, Kühne M, Heckmann M, Fleckenstein J, et al. (2005) In vivo formation and repair of DNA double-strand breaks after computed tomography examinations. Proc Natl Acad Sci U S A 102: 8984-8989.

[36] Haddock MG, Swaminathan R, Foster NR, Hauge MD, Martenson JA, et al. (2007) Gemcitabine, cisplatin, and radiotherapy for patients with locally advanced pancreatic adenocarcinoma: results of the North Central Cancer Treatment Group Phase II Study N9942. J Clin Oncol 25:2567-2572. 